\title{
Producing GHZ state of nitrogen-vacancy centers in cavity QED
}

\author{
Zheng-Yuan Xue and Sheng Liu \\ Laboratory of Quantum Information Technology, and School of Physics and Telecommunication Engineering, \\ South China Normal University, Guangzhou 510006, China
}

(Dated: September 1, 2018)

\begin{abstract}
We propose a scheme to generate GHZ state of nitrogen-vacancy centers coupled to a whispering-gallery mode cavity. The implemented evolution is independent on the cavity field state with the assistant of a strong classical field, and thus not sensitive to the thermal state of the cavity. Meanwhile, it is fast compare to the convectional dispersive interaction induced operation in a cavity-assisted system. The scheme is readily scalable to multiqubit scenario.
\end{abstract}

PACS numbers: 03.67.Lx, 42.50.Dv

Keywords: cavity QED; GHZ state; Nitrogen-vacancy centers

Entanglement is one of the most counterintuitive properties of quantum mechanics, which plays an central role in quantum information and quantum computation [1]. For real quantum system, it is generally sensitive to practical noise, which results from the infamous decoherence effect. Therefore, generation of entangled state with inherent robustness to diverse noise is preferred. Due to long electronic spin lifetime, fast initialization, good qubit readout, and coherent manipulation at room temperature, the diamond nitrogen-vacancy (NV) center is considered as a promising candidates for quantum computing [2]. Coupling of many qubits in solid state systems is problematic. For NV center, the partial solution is introduce coherent coupling between electron and nuclear spin qubits. However, this can only be done in the case of a few qubits since there are limited number of nuclear spins can be individually addressed [3]. Alternatively, separated NV centers can be coupled by a cavity mode [4]. For a easy fabricated microsphere cavity, the quantized whispering-gallery mode (WGM) may has a ultrahigh Q factor and very small volume [5], and thus leading to reach the strong coupling regime. Entanglement generation schemes are also proposed with a microsphere cavity [6] or a one dimensional resonator [7]. However, the previous schemes all work in the dispersive regime, i.e., the detuning between the cavity mode frequency and the qubits is much larger than that of the cavity-qubit interaction, which makes the effective coupling relatively weaker compare to resonant coupling, that is, the generation time will be longer. Meanwhile, the coupling usually contains cavitystate-dependent energy shift, which leads the generation to be sensitive to the cavity thermal state.

Here, we propose an alternative scheme for generating multipartite entangled state of nitrogen-vacancy centers coupled to a whispering-gallery mode cavity, which overcome the above-mentioned drawbacks. In addition to the optical transitions driven by the cavity mode and optical laser, we introduce another transition within the ground states strongly driven by a microwave field. Before proceeding, we want to emphasize that using both optical and microwave radiation to control an electron spin associated with the NV center in diamond is experimentally accessible [8]. With the driven of a microwave field, one get two additional merits of the proposed scheme.
Firstly, the photon-number-dependent parts in the evolution operator are canceled, and thus the scheme is insensitive to the thermal state of the cavity mode. Secondly, the large detuning constrain can be removed so that fast gate operation can be achieved. Meanwhile, the solid-state set-up is readily scalable to multiqubit scenario and the gate speed is not slowed down with the increasing of the involved qubits.

As illustrated in Fig. 1(a), $N$ identical NV centers in $N$ separate diamond nanocrystals may be strongly coupled to the WGM of a microsphere cavity. The lowest-order WGM corresponding to the light traveling around the equator of the microsphere, which offers exceptional mode properties for reaching strong coupling regime. One can model the NV center as a three-level system, as shown in Fig. 1(b), where the states $\left|{ }^{3} A, m_{s}=0\right\rangle$ and $\left|{ }^{3} A, m_{s}=-1\right\rangle$ are encoded as our qubit states $|0\rangle$ and $|1\rangle$, respectively. The state $\left|{ }^{3} E, m_{s}=0\right\rangle$ is labeled by $|e\rangle$ and do not use the metastable ${ }^{1} A$ state, which has not yet been fully understood [9]. In our implementation, the optical transition $|0\rangle \rightarrow|e\rangle$ and $|1\rangle \rightarrow|e\rangle$ (with transition frequencies $\omega_{e 0}$ and $\omega_{e 1}$ ) are coupled by the WGM with frequency $\omega_{c}$ and a laser with frequency $\omega_{L}$ and polarization $\sigma^{+}$[10], respectively. Both coupling is far-off resonant from their transition frequencies so that the $|e\rangle$ state can be adiabatically eliminated. The NV centers are fixed and apart with the distance much larger than the wavelength of the WGM, so that they can interact individually with the laser beams and the direct coupling among them is negligible. Then, in units of $\hbar=1$, the system is described by

$$
\begin{aligned}
H_{S}= & \omega_{c} a^{+} a+\sum_{i} \omega_{i}|i\rangle\langle i| \\
& +\sum_{j=1}^{N}\left[G_{j} a|e\rangle\left\langle 0\left|+\Omega_{L, j} e^{-i\left(\omega_{L}+\phi\right)}\right| e\right\rangle\langle 1|+\text { H.c. }\right],
\end{aligned}
$$

where $a^{+}(a)$ is the creation (annihilation) operator of the WGM field, $\omega_{i}$ is the frequency of $i$ th energy level with $i \in\{0,1, e\}, G_{j}$ and $\Omega_{L, j}$ is the coupling strength between $j$ th NV center and WGM and laser, respectively.

Using the rotating-wave approximation (RWA), with respect to the free Hamiltonian $H_{0}=\omega_{c} a^{+} a+\sum_{i} \omega_{i}|i\rangle\langle i|$, the interaction Hamiltonian in the interaction picture can be 
(a)

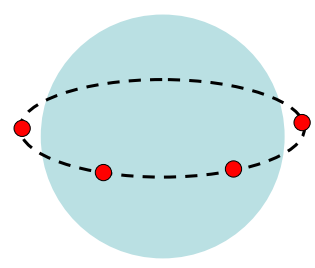

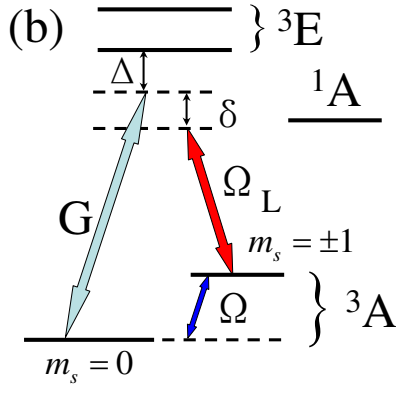

FIG. 1: (a) Schematic setup of the microsphere cavity, where $N$ identical NV centers (red dots) in diamond nanocrystals are equidistantly attached around the equator of a single fused-silica microsphere cavity. (b) Level diagram for a NV center, with $G$ and $\Omega_{L}$ are the coupling strength between NV center and WGM and the laser pulse, respectively. We encode qubits in the subspace spanned by the state of $m_{s}=0$ and $m_{s}=-1$. The qubit zero-field splitting is $\omega_{10}=2.88$ $\mathrm{GHz}$ and can be driven by a microwave field with strength $\Omega$.

written as [11]

$$
H_{I}=\sum_{j=1}^{N} \eta_{j}\left(a \sigma_{j}^{+} e^{-i\left(\delta_{j} t-\phi\right)}+\text { H.c. }\right)
$$

where $\sigma_{j}^{+}=\left|1_{j}\right\rangle\left\langle 0_{j}\left|, \sigma_{j}^{-}=\right| 0_{j}\right\rangle\left\langle 1_{j}\right|$, and $\eta_{j}=$ $G_{j} \Omega_{L, j}\left(\frac{1}{\Delta_{j}+\delta_{j}}+\frac{1}{\Delta_{j}}\right)$ with $\Delta_{j}=\omega_{e 0, j}-\omega_{c}$ and $\delta_{j}=$ $\omega_{c}-\omega_{10, j}-\omega_{L, j}$. For simplicity, hereafter, we assume that $\eta_{j}=\eta$ and $\delta_{j}=\delta$. However, this is not necessary here. The inhomogeneous qubit-cavity coupling will result in deviation of the generated final state from the target state by extra phase factor, which can be easily compensated by single qubit operations [12]. Meanwhile, besides the optical coupling, we also add a resonant microwave driven field at a frequency of the microwave transition $\omega_{10}$ for each NV center. Then, in the above interaction picture, it reduce to $H_{d}=\frac{\Omega}{2} \sigma_{j}^{x}$ with the Pauli matrix written in the qubit subspace. Then the total interaction Hamiltonian can be written as

$$
H_{1}=\sum_{j=1}^{N}\left[\frac{\Omega}{2} \sigma_{j}^{x}+\eta\left(a \sigma_{j}^{+} e^{-i(\delta t-\phi)}+a^{+} \sigma_{j}^{-} e^{i(\delta t-\phi)}\right)\right] .
$$

This microwave control over the NV centers is well studied experimentally [13] and even all the three magnetic sub-levels can be resolved [14]. With this strong microwave coupling, we can realize the analogy of strong-driven atomic cavity QED system with two level atoms [15]. Meanwhile, this distinct our scheme with all the previous investigation with $\mathrm{NV}$ centers coupled by a cavity mode [4, 6, 7]. In the interaction picture with respect to the first term, the interacting Hamiltonian reads [15]

$$
H_{2}=\frac{\eta}{2} a e^{-i(\delta t-\phi)} \sum_{j=1}^{N}\left(\widetilde{\sigma}_{z}^{j}+e^{i \Omega t}|+\rangle_{j}\left\langle-\left|-e^{-i \Omega t}\right|-\right\rangle_{j}\langle+|\right)
$$

+ H.c. where $\widetilde{\sigma}_{z}$ denotes Pauli matrix in the eigen basis of $\sigma_{x}$ with eigen equations as $\sigma_{x}| \pm\rangle= \pm| \pm\rangle$, where $| \pm\rangle=(|0\rangle \pm$ $|1\rangle) / \sqrt{2}$. In the case of $\Omega \gg\{\delta, \eta\}$, we can omitting the fast oscillation terms with frequencies $\Omega \pm \delta$ using RWA, then the Hamiltonian (4) reduces to

$$
H_{3}=\eta\left(a e^{-i(\delta t-\phi)}+a^{\dagger} e^{i(\delta t-\phi)}\right) J_{x},
$$

where $J_{x}=\sum_{j=1}^{N} \sigma_{j}^{x} / 2$. For $\phi=0$, the above Hamiltonian reduces to

$$
H_{D 1}=\eta\left(a e^{-i \delta t}+a^{\dagger} e^{i \delta t}\right) J_{x} .
$$

The time-evolution operator for the Hamiltonian in Eq. (6) can be expressed as [16-19]

$$
\begin{aligned}
U(t)= & \exp \left[-i A(t) J_{x}^{2}\right] \\
& \times \exp \left[-i B(t) a J_{x}\right] \exp \left[-i B^{*}(t) a^{\dagger} J_{x}\right],
\end{aligned}
$$

where

$$
\begin{gathered}
A(t)=\frac{\eta^{2}}{\delta}\left[\frac{1}{i \delta}\left(e^{i \delta t}-1\right)-t\right], \\
B(t)=i \frac{\eta}{\delta}\left(e^{-i \delta t}-1\right) .
\end{gathered}
$$

While the whole time evolution is nonperiodic, Eq. (9) shows that $B(t)$ is a periodic function of time, and it vanishes at $\delta T=2 k \pi$ where $k=1,2,3, \ldots$. At these times, the time evolution operator in the interaction picture reduces to

$$
U\left(\gamma^{\prime}\right)=\exp \left(i \gamma^{\prime} J_{x}^{2}\right),
$$

with $\gamma^{\prime}=\eta^{2} T / \delta \equiv \lambda T$.

The operator in Eq. (10) is readily to be used for generating GHZ state. However, this requires a carefully chosen duration of the interaction because it involves real excitation of the cavity photon. Fortunately, idea similar to the spin echo and dynamical decoupling can be used to optimize the generation process [20-22]. The key is the map of $H_{D 1} \rightarrow H_{D 2}=-H_{D 1}$, which can be conveniently achieved by setting $\phi=\pi$ in our scheme. Then, a gate

$$
U(\gamma)=\exp \left(i \gamma J_{x}^{2}\right)
$$

with $\gamma=\frac{\eta^{2}}{\delta}\left(\frac{2}{\delta} \sin \frac{\delta t}{2}-t\right)$ can be obtained as the following [20-22]. For a gate with duration $t$, one can set $H_{D 1}$ for the duration of $0 \sim t / 2$ and $H_{D 2}$ for the left duration $t / 2 \sim t$ [20], similar to the dynamic decoupling method. Alternatively, the map of $H_{D 1} \rightarrow-H_{D 1}$ can be done by $\pi$ pulses on the qubits [12], similar to the spin echo method.

It is notable that $U(\gamma)$ operation proposed here possess the following distinct merits. Firstly, it is insensitive to the thermal state of the cavity mode as the related influence represented by the last two exponents in Eq. (7) is completely removed. Secondly, it also remove the constrain of large detuning. It is noted that $T \sim 1 / \eta$ for $\delta \sim \eta$, which is comparable 
to the resonant coupling strategy and is much faster than that of the conventional dispersive coupling with $\delta \gg \eta$. This fast gate operation is very important in view of limited coherence times. Thirdly, it is obvious that the time needed for this gate operation is comparable to a two-qubit gate as $A(t)$ is independent on $N$, and thus the gate speed is not slowed down with the increasing of qubits. Therefore, it is readily scalable to multiqubit scenario.

Now, we illustrate how to generate the GHZ state in this system, which has many application in quantum communication [23]. By choosing $\gamma=\pi / 2$ and an initial state $|\Psi\rangle_{i}=|00 \cdots 0\rangle$ for $N$ NV centers, the final state $|\Psi\rangle_{f}=$ $\exp \left(i \frac{\pi}{2} J_{x}^{2}\right)|\Psi\rangle_{i}$ is found to be a GHZ state given by [19]

$$
\left|\Psi^{f}\right\rangle=\frac{1}{\sqrt{2}}\left[e^{-i \frac{\pi}{4}}|00 \cdots 0\rangle+e^{i \pi\left(\frac{1}{4}+\frac{N}{2}\right)}|11 \cdots 1\rangle\right],
$$

when $N$ is even. Note that $\gamma=\pi / 2$ can be achieved when choosing the parameter as $\delta=2 \sqrt{k} \eta$. Then, for $k=1$, one obtains $\delta=2 \eta$ and the entanglement generation time as $T=$ $\pi / \eta$. For odd $N$, one can get GHZ state by applying another unitary operator $U=\exp \left(-i \frac{\pi}{2} J_{x}\right)$ in addition to Eq. (11).

In the derivation of effective Hamiltonian in Eq. (5), we have neglected the following terms

$$
\begin{aligned}
H_{n} & =\frac{\eta}{2} a e^{-i \delta t} \sum_{j=1}^{N}\left(e^{i \Omega t}|+\rangle_{j}\left\langle-\left|-e^{-i \Omega t}\right|-\right\rangle_{j}\langle+|\right)+\text { H.c. } \\
& =\frac{i \eta}{2} a e^{-i \delta t} \sum_{j=1}^{N}\left[\sin (\Omega t) \sigma_{j}^{z}-\cos (\Omega t) \sigma_{j}^{y}\right]+\text { H.c., (13) }
\end{aligned}
$$

which could reduce the fidelity of the generated state. However, for $\sigma_{z}$ terms, one can use the spin-echo technique to eliminate the infidelity [12]. Following Ref. [19], we consider the effect of the $\sigma_{y}$ terms on the gate operation. In the interaction picture with respect to our free Hamiltonian $H_{\text {free }}=\delta a^{+} a+\sum_{j=1}^{N} \frac{\Omega}{2} \sigma_{j}^{x}$, the interaction Hamiltonian is $H_{n, I}(t)=U^{\dagger}(t) H_{n} U(t)$ with $U(t)=\exp \left(-i H_{\text {free }} t\right)$, and we have the propagator $U^{\prime}(t)$ from the Dyson series. During the integration, one can treat $U(t)$ as a constant as $H_{n}(t)$ is oscillating much faster than the propagator. Then, under this assumption, we get

$$
\begin{aligned}
U^{\prime}(t)= & 1-\frac{\eta}{2 \Omega} \sin (\Omega t) \sum_{j=1}^{N} U^{\dagger}(t) \sigma_{j}^{y} U(t) \\
& \left.+i \frac{\eta^{2}}{4 \Omega^{2}} \sum_{k \neq j}^{N}[1-\cos (2 \Omega t)] U^{\dagger}(t) \sigma_{j}^{y} \sigma_{k}^{y} U(t)\right\}+\cdots
\end{aligned}
$$

At the time $\delta t=2 n \pi$, the infidelity is

$$
F_{\text {in }}=\xi[1-\cos (2 \Omega t)],
$$

where $\xi=N(N-1) \eta^{2} /\left(8 \Omega^{2}\right)$. Noted that the estimation of $F_{i n}$ is obtained in the interaction picture. Return to the rotating frame, the time evolution operator will get the following additional term of $U_{x}=\exp \left(-i \sum_{j=1}^{N} \frac{\Omega}{2} \sigma_{j}^{x} t\right)$. Therefore, control the duration of classical microwave field accurately to fulfill $\Omega t=4 n \pi$, the effect of the $\sigma_{y}$ terms and $U_{x}$ will both vanishes. Note that $\delta t=2 n \pi$, so $\Omega / \delta=2 n$. Considering the further requirement of $\Omega \gg \delta$, we may choose $\Omega=6 \delta$.

To see the feasibility of the present scheme with current technology, we now use typical values of physical parameters in the system to estimate the operation time $T$. NV centers are located near the microcavity surface in order make the coupling between the NV center and WGM maximum, which could be $G_{\max } \simeq 2 \pi \times 1 \mathrm{GHz}$ with the transition wavelength between the states $|e\rangle$ being $637 \mathrm{~nm}$, the spontaneous decay rate of the excited state being $\Gamma_{0}=2 \pi \times 83 \mathrm{MHz}$, and the mode volume be $V_{m}=100 \mu m^{3}$ [24]. For $\delta \ll \Delta$, $\eta \simeq 2 G \Omega_{L} / \Delta=2 \pi \times 50 \mathrm{MHz}$, with $\Omega_{L}=2 \pi \times 500 \mathrm{MHz}$ and $\Delta=2 \pi \times 20 \mathrm{GHz}$. The effective qubit spontaneous emission rate is estimated as [25] $\Gamma_{\text {eff }}=\Gamma_{0} \Omega_{L} G / \Delta^{2} \approx 2 \pi \times 0.1$ MHz. In the fused-silica microsphere cavity, the small radius of $10 \mu \mathrm{m}$ could lead to the $Q$ factor exceeding $10^{9}$ with possible improvement as demonstrated in [26]. The cavity decay rate rate is $\kappa=\omega_{c} / Q=2 \pi \times 0.47 \mathrm{MHz}$ for $Q=10^{9}$. In our scheme, for $\delta=2 \eta=2 \pi \times 100 \mathrm{MHz}$, the assumption $\delta \ll \Omega$ with $\Omega=2 \pi \times 600 \mathrm{MHz}$ [13] and $\delta \ll \Delta$ is well-satisfied, then the entangled state generation time is $T=10 \mathrm{~ns}$, which is negligible small compare to both the cavity decay and qubit coherence time even at room temperature.

With the above parameters, for $N=4, \xi \approx 0.01$, and thus $F_{\text {in }}<2.1 \%$, which increases with the increasing of $N$. To see the time dependence of the fidelity of the generated entangled state, as shown in Fig. (2), we numerically simulated the time-evolution of the effective Hamiltonian of Eq.
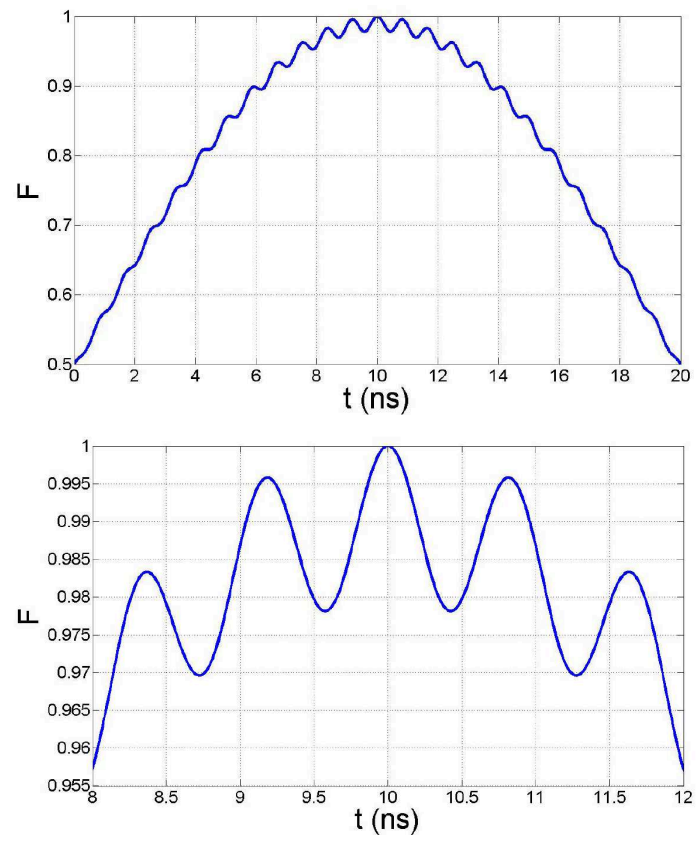

FIG. 2: Fidelity of the entanglement generation, see the main text for the chosen parameters. 
(11) plus $H_{n}$ in Eq. (14). We have defined the fidelity as $F=\left\langle\Psi^{f}|U(\gamma)| \Psi^{f}\right\rangle \times\left(1-F_{i n}\right)$. From the lower one of Fig. (2), we can see that the generation is now very robust to small deviation of the time duration.

In summary, we have proposed a scheme to generate GHZ state of nitrogen-vacancy centers coupled to a whisperinggallery mode cavity. The fast scheme is not sensitive to both the spontaneous emission decay of the NV centers and thermal state of the cavity. Finally, the scheme is readily scalable to multiqubit scenario as the generation time is independent on the number of involved qubits.

This work was supported by the NFRPC (No. 2013CB921804), the NSFC (No. 11004065), the PCSIRT, and the NSF of Guangdong province (No. S2011040000403).

[1] M.A. Nielsen and I.L. Chuang, Quantum Computation and Quantum Information (Cambridge University Press, Cambridge, 2000).

[2] E. Togan, Y. Chu, A. S. Trifonov, L. Jiang, J. Maze, L. Childress, M. V. G. Dutt, A. S. Søensen, P. R. Hemmer, A. S. Zibrov, and M. D. Lukin, Nature (London) 466, 730 (2010).

[3] P. Neumman, N. Mizuochi, F. Rempp, P. Hemmer, H. Watanabe, S. Yamasaki, V. Jacques, T. Gaebel, F. Jelezkoand, and J. Wrachtrup, Science 320, 1326 (2008).

[4] Y.-S. Park, A. K. Cook, and H. Wang, Nano Lett. 6, 2075 (2006); M. Larsson, K. N. Dinyari, and H. Wang, Nano Lett. 91447 (2009).

[5] D. K. Armani, T. J. Kippenberg, S. M. Spillane, and K. J. Vahala, Nature (London) 421, 925 (2003).

[6] W. L. Yang, Z. Q. Yin, Z. Y. Xu, M. Feng, and J. F. Du, Appl. Phys. Lett. 96241113 (2010); W. L. Yang, Z. Y. Xu, M. Feng, and J. F. Du, New J. Phys. 12, 113039 (2010).

[7] Z. Y. Xu, Y. M. Hu, W. L. Yang, M. Feng, and J. F. Du, Phys. Rev. A 80, 022335 (2009); Q. Chen, Z. Y. Xu, and M. Feng, Phys. Rev. A 82, 014302 (2010); W. L. Yang, Y. Hu, Z. Q. Yin, Z. J. Deng, and M. Feng, Phys. Rev. A 83, 022302 (2011).

[8] M. V. Gurudev Dutt, L. Childress, L. Jiang, E. Togan, J. Maze, F. Jelezko, A. S. Zibrov, P. R. Hemmer, and M. D. Lukin, Sci- ence 316, 1312 (2007)

[9] N. B. Manson, J. P. Harrison, and M. J. Sellars, Phys. Rev. B 74, 104303 (2006).

[10] C. Santori, P. Tamarat, P. Neumann, J. Wrachtrup, D. Fattal, R. G. Beausoleil, J. Rabeau, P. Olivero, A. D. Greentree, S. Prawer, F. Jelezko, and P. Hemmer, Phys. Rev. Lett. 97, 247401 (2006).

[11] K.-H. Song, Z.-W. Zhou, and G.-C. Guo, Phys. Rev. A 71, 052310 (2005); D. F. V. James and J. Jerke, Can. J. Phys. 85, 625 (2007).

[12] D. Leibfried, B. DeMarco, V. Meyer, D. Lucas, M. Barrett, J. Britton, W. M. Itano, B. Jelenkovic, C. Langer, T. Rosenband, and D. J. Wineland, Nature 422, 412 (2003).

[13] G. D. Fuchs, V. V. Dobrovitski, D. M. Toyli, F. J. Heremans, and D. D. Awschalom, Science 326, 1520 (2009).

[14] F. Shi, X. Rong, N. Xu, Y. Wang, J. Wu, B. Chong, X. Peng, J. Kniepert, R.-S. Schoenfeld, W. Harneit, M. Feng, and J. Du, Phys. Rev. Lett. 105, 040504 (2010).

[15] E. Solano, R.L. de Matos Filho, and N. Zagury, Phys. Rev. Lett. 90, 027903 (2003).

[16] S.-B. Zheng, Phys. Rev. A 68, 035801 (2003).

[17] S.-L. Zhu, Z. D. Wang, and P. Zanardi, Phys. Rev. Lett. 94, 100502 (2005).

[18] Z.-Y. Xue and Z. D. Wang, Phys. Rev. A 75, 064303 (2007); Z.-Y. Xue, Quantum Inf. Process. 11, 1381 (2012).

[19] A. Søensen, and K. Mømer, Phys. Rev. A 62, 022311 (2000); K. Mømer and A. Søensen, Phys. Rev. Lett. 82, 1835 (1999).

[20] Y.-D. Wang, A. Kemp, and K. Semba Phys. Rev. B 79, 024502 (2009).

[21] W.-P. Zou, G. Zhang, and Z.-Y. Xue, Chin. Phys. Lett. 28, 120308 (2011).

[22] D. Hayes, S. M. Clark, S. Debnath, D. Hucul, I.V. Inlek, K.W. Lee, Q. Quraishi, and C. Monroe, Phys. Rev. Lett. 109, 020503 (2012).

[23] Z.-Y. Xue, Y.-M. Yi, and Z.-L. Cao, J. Mod. Opt. 53, 2725 (2006); Chin. Phys. 15, 1421 (2006); Physica A 374, 119 (2007).

[24] P. Neumann, R. Kolesov, V. Jacques, J. Beck, J. Tisler, A. Batalov, L. Rogers, N. B. Manson, G. Balasubramanian, F.Jelezko, and J.Wrachtrup, New. J. Phys. 11, 013017 (2009).

[25] S. G. Clark and A. S. Parkins, Phys. Rev. Lett. 90, 047905 (2003).

[26] D. W. Vernooy, V. S. Ilchenko, H. Mabuchi, E. W. Streed, and H. J. Kimble, Opt. Lett. 23, 247 (1998). 